\title{
Evolving Role of Genomics in Genitourinary Neoplasms
}

\author{
Michael E. Devitt, Robert Dreicer
}

Department of Medicine, Division of Hematology/Oncology, University of Virginia School of Medicine

\author{
Correspondence: \\ michaeldevitt@virginia.edu \\ Tel.: + 14349823209 \\ Fax.: + 4342447534
}

Received: 4 September 2018

Accepted: 8 February 2019

Key Words: Biomarkers • Risk - Prostate Cancer - Renal Cell Carcinoma • Urothelial Carcinoma.

\begin{abstract}
The aim of this article is to review the current role of genomic testing in the risk, prognosis, and treatment of genitourinary malignancies. The authors selected guidelines, publications, and abstracts relevant to the current and emerging role of genomics in genitourinary cancers. The risk of developing genitourinary cancer can be stratified based on genomic data. Prostate cancer has the strongest degree of heritability, with $B R C A 1 / 2$ and $H O X B 13$ mutations playing a role in familial disease. Genomic data is on the verge of informing treatment decisions across genitourinary cancers. mCRPC has diverse genomic alterations that represent potential therapeutic targets, including alterations in the AR pathway, DNA damage and repair pathways, cell cycle pathways, PI3K pathway, and Wnt signaling. Genomic alterations in clear cell renal cell carcinoma can inform prognosis and mutations in mTOR pathways predict response to mTOR inhibitors. Urothelial carcinoma can be classified into different subtypes based on gene expression profiling, which provides prognostic information and predicts response to chemotherapy and immunotherapy. Specific mutations have been identified that predict response to therapy including ERCC2 mutations and cisplatin, DNA damage and repair mutations and checkpoint inhibitors, and FGFR3 mutations and FGFR tyrosine kinase inhibitors such as erdafitinib. Conclusion. Genitourinary malignancies have not felt the impact of genomic data as greatly as other cancer types. The majority of benefit lies in identifying patients at high risk of genitourinary cancer. Fortunately, breakthroughs are on the horizon that will result in a greater incorporation of genomic information into treatment decisions for patients with genitourinary cancer.
\end{abstract}

\section{Introduction}

Genomic testing has altered the practice of oncology, informing multiple facets of the care of patients with a variety of neoplasms. The myriad of publications from the Human Genome project has led to a paradigm shift and changes in our ability to correlate genetic information with biologic behavior. Rapid developments in technology resulting in more cost effective and faster sequencing of genetic information, led to the swift, albeit still evolving integration of this information into clinical practice. Genetic information may help identify populations at a high risk of genitourinary malignancies and can increasingly inform treatment decisions.

In this review, we aim to provide an overview of the role of genomic testing in the care of patients with genitourinary malignancies, focusing on prostate, kidney, and urothelial cancers, and touch on some of the emerging applications of genomics that may evolve into standards of care. 


\section{Prostate Cancer}

\section{Genomics Inform Risk}

Prostate cancer represents one of the most heritable forms of malignancy. The Nordic Twin Study of Cancer showed an increase in the relative risk of developing prostate cancer of up to 5.69 times and 1.7 times for monozygotic and dizygotic twins respectively $(1,2)$. Men with a positive family history of prostate cancer in a first degree relative are at higher risk of prostate cancer, which further increases if more than one first degree relative is affected (3). Mutations in the genes homeobox B13 (HOXB13) and breast cancer susceptibility types 1 and 2 (BRCA1/2) have been associated with familial clusters of prostate cancer. HOXB13 is a tumor suppressor gene that encodes for a DNA-binding domain. The mutation G48E in HOXB13 was associated with familial prostate cancer in 2012 (4) and has subsequently been shown to confer an approximately 4.5 fold increased risk in the development of prostate cancer for carriers of the mutation (5). BRCA1/2 encode for DNA repair machinery and have been implicated in the hereditary breast and ovarian cancer (HBOC) syndrome. Men carrying mutations in BRCA1 or BRCA2 have an increased risk of developing prostate cancer by up to 3.8 fold and 8.6 fold respectively (6). In addition, BRCA 2 mutations have been associated with an earlier onset of prostate cancer $(7,8)$ and both BRCA1 and BRCA2 mutations with more aggressive clinicopathologic disease $(9,10)$. The DNA mismatch repair proteins encoded by the genes $\mathrm{MSH} 2$, $M L H 1$, and MSH6, have also been associated with an increased risk of prostate cancer in the Danish HNPCC registry (11). However, these genes account for a small proportion of the heritable risk. Genome wide association studies have shown nearly 170 single nucleotide polymorphisms (SNPs) that are associated with an increased risk of prostate cancer (12). No individual SNP variant carries substantial risk on its own to be of clinical utility.

Men with a known family history of Lynch syndrome or mutation in HOXB13 or $B R C A 1 / 2$ should be informed of the risks associated with these mutations and the utility of germline testing if they do not have prostate cancer. This may provide information relevant to the risk and benefit counseling regarding the utility of prostate specific antigen (PSA) screening. A family history suggestive of the HBOC syndrome should also prompt as discussion regarding the utility of germline testing for the presence of $B R C A 1 / 2$, preferably with a genetic counselor when available (13).

\section{Clinical Decision Making}

Genomic testing can help inform prognosis and treatment in patients with an established diagnosis of prostate cancer. The utility of genomic testing has been primarily shown in the metastatic castrate-resistant prostate cancer (mCRPC). mCRPC harbors a multitude of genomic variations. Mutations affecting the androgen receptor pathway, DNA mismatch repair machinery, cell cycle pathway, phosphatidylinositol3-kinase (PI3K) pathway, and Wnt signaling have been identified (14). Therapeutic agents targeting each of these pathways have been developed or are in development and several are being tested in prostate cancer.

Mutations in the androgen receptor (AR) pathway are present frequently in prostate cancer, occurring in over $70 \%$ of mCRPC cases in a series of 150 patients (14), confirming the importance of the AR pathway in the pathogenesis of prostate cancer. This dependence on AR signaling, even in the advanced castrate-resistant state, establishes a biologic rationale for the efficacy of next generation anti-androgens such as abiraterone acetate and enzalutamide. Ge- 
nomic alterations in the AR pathway can lead to resistance to these agents. AR-V7 is a splice variant of the androgen receptor that leads to resistance to abiraterone and enzalutamide and develops in nearly a third of men treated with these agents (15). Recently presented data from the prospective, multicenter PROPHECY trial showed that patients with AR-V7 positivity determined by circulating tumor cells were unlikely to respond to abiraterone or enzalutamide and had shortened progression free survival and overall survival (16). AR-V7 does not confer resistance to non-AR targeted therapies and there is evidence that treatment with docetaxel may eliminate AR-V7 clones (17). Whether this can re-sensitize a patient to AR-targeted therapy remains in question.

Mutations in DNA-repair genes have been identified as an important and potentially under-identified contributor to prostate cancer. In mCRPC, mutations in DNA repair may be present in up to $22.7 \%$ of patients (14). More importantly, germline mutations in these genes are fairly common and under-identified. In a population of $692 \mathrm{men}$ with metastatic prostate cancer, germline mutations in the DNA-repair genes $B R C A 2$, ATM, CHEK2, BRCA1, RAD51D, and $P A L B 2$ were identified in $11.8 \%$ of patients (18). Current guidelines recommend testing men for the presence of $B R C A 1 / 2$ mutations in men with prostate cancer with a Gleason score of 7 or greater and one of the following: close relative with ovarian or breast cancer diagnosed at age 50 or younger; or at least two relatives with breast, ovarian, or prostate cancer (Gleason 7 or greater) at any age (13). However, recent data suggests that as many as $40 \%$ of men with prostate cancer and germline mutations do not meet criteria for testing under the current guidelines (19), which likely contributes to the under-recognition of these germline abnormalities. In addition, guidelines only focus on the testing for $B R C A 1 / 2$ and do not include recom- mendation regarding broad testing for the other potential germline mutations. Several classes of therapeutics have potential activity in patients with defective DNA repair genes, including immunotherapy, PARP inhibitors, and platinum chemotherapy. Defects leading to microsatellite instability represent a prostate cancer population that has a much greater chance of response to immune checkpoint inhibitors but are found in $<2.5 \%$ of patients (20). Patients with Lynch syndrome associated prostate cancers have a higher likelihood of microsatellite instability (11). Platinum-based chemotherapy has also been associated with improved responses in patients with DNA repair defects in the BRCA2 gene (21). The role of PARP inhibitors in the treatment of prostate cancer is an area of active research. Early data showed that in patients with known defects in DNA repair machinery who had progressed on standard therapies, olaparib elicited an overall response rate of 33\% with response defined as either PSA decline of $50 \%$ or greater, radiographic response by RECIST 1.1, or a reduction in volume of circulating tumor cells (22). Multiple phase III trials investigating the role of PARP inhibitors in $\mathrm{MCRPC}$ are ongoing, both in selected and unselected patient populations, as well as single agent and combination therapy.

PI3K pathway mutations may be present in almost $50 \%$ of mCRPC (14). The mutated genes in this pathway included PTEN, PIK$3 C A, A K T 1$, and PIK3CB. The use of PI3K inhibitors has been studied in unselected patients with mCRPC. Buparlisip, a pan-class I PI3K inhibitor, did not yield PSA responses in men with $\mathrm{mCRPC}$ who had progressed on docetaxel and/or enzalutamide in a single arm phase II trial (23). In a small phase I trial, a novel inhibitor of $\mathrm{PI} 3 \mathrm{~K} \beta$ showed some evidence of activity in MCRPC patients whose tumors harbored $P I 3 K C B$ abnormalities (24). This is an emerging area of research. 


\section{Summary}

Genomic alterations have become increasingly important to our understanding of the biology of prostate cancer (Table 1). Genetics clearly play a role in an individual's risk of developing prostate cancer, with connections between mutations in HOXB13 and $B R C A 1 / 2$ to familial clusters of prostate cancer. For men who have a diagnosis of mCRPC, the presence of mutations in DNA repair genes is under-recognized and is clinical useful. Standard therapies such as platinum chemotherapy and immunotherapy for MSI-high tumors can be considered in patients harboring DNA repair abnormalities. Additional mutations are on the cusp of demonstrating clinical utility and many future therapies will likely be dependent on the presence of genomic biomarkers. Ongoing research is needed to further define optimal use of germ-line and somatic testing of patients with advanced prostate cancer.

\section{Kidney Cancer}

\section{Genetic Risk}

The Nordic Twin Study of Cancer suggested that kidney cancer showed evidence of heritability, though the familial risk estimation was quite low in dizygotic twins (1). Several genes have been implicated in inherited syndromes that are associated with an increased risk of developing kidney cancer (Table 2). These inherited syndromes represent a small proportion of newly diagnosed renal cancers, but have informed our understanding of the biology of kidney cancer. Unfortunately, no established screening paradigm exists for kidney cancer. Patients with a known inherited syndrome are frequently recommended, based on expert opinion, to undergo annual cross sectional imaging for surveillance with either computed tomography or magnetic resonance imaging of the abdomen.

Table 1. Prostate Cancer Summary

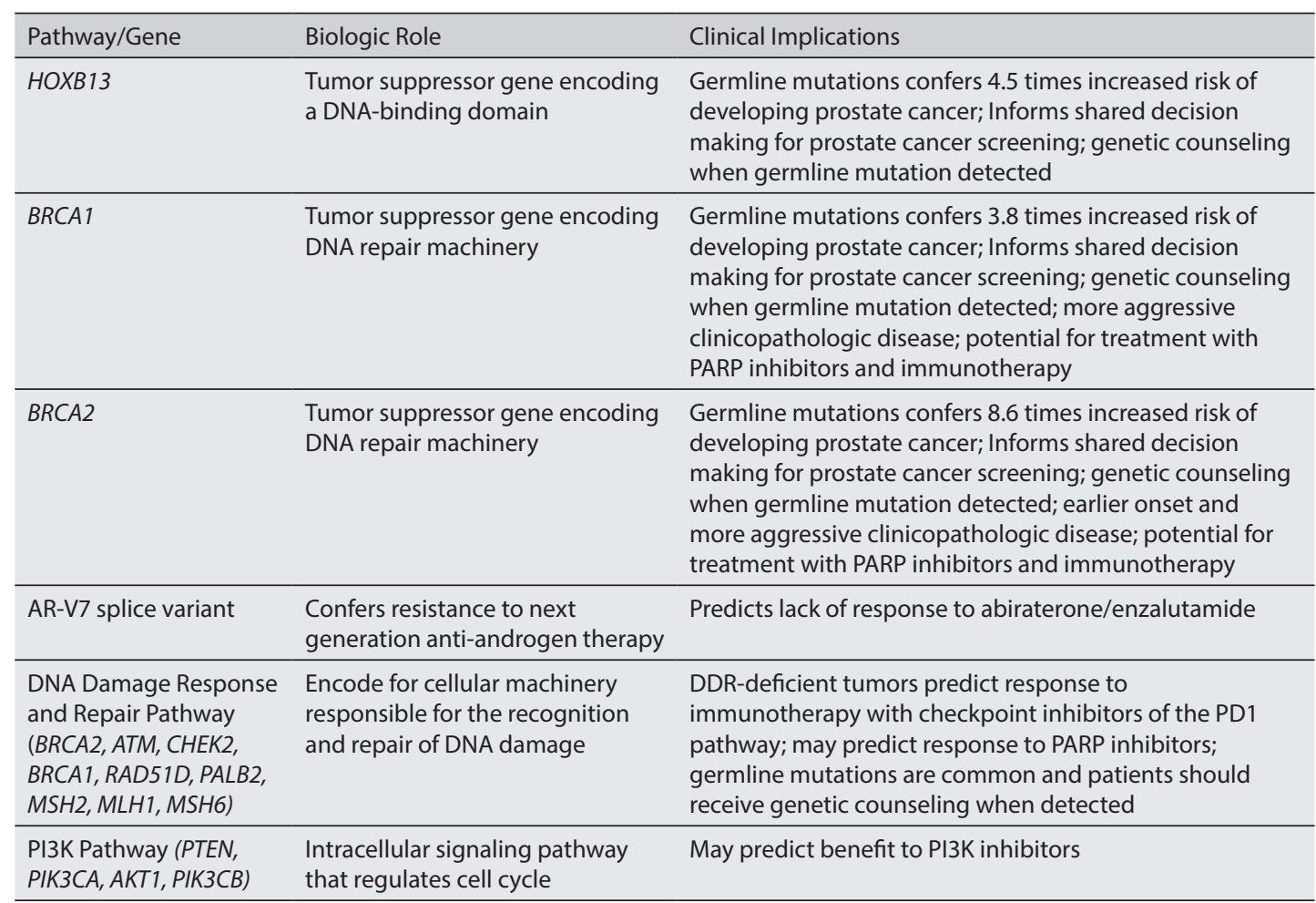


Acta Medica Academica 2019;48(1):68-77

Table 2. Syndromes Associated with Increased Risk of Renal Cell Carcinoma

\begin{tabular}{|c|c|c|}
\hline Syndrome & Mutation & Clinical Features \\
\hline \multicolumn{3}{|l|}{ Clear Cell Carcinoma } \\
\hline Von Hippel Lindau & VHL & $\begin{array}{l}\text { CNS hemangioblastomas, pheochromocytoma, pancreatic } \\
\text { neuroendocrine tumor }\end{array}$ \\
\hline PTEN Hamartoma Syndrome & PTEN & $\begin{array}{l}\text { Lipomas, fibromas, acral keratosis, } \\
\text { Gl polyps; increased risk of cancers of the breast, thyroid, and } \\
\text { endometrium; papillary renal carcinoma also seen }\end{array}$ \\
\hline $\begin{array}{l}\text { Familial Clear Cell with } \\
\text { Chromosome } 3 \text { Translocation }\end{array}$ & $\begin{array}{l}\text { Translocation } \\
\text { chromosome } 3\end{array}$ & Clear cell kidney cancer \\
\hline BAP1 Mutant Disease & BAP1 & Melonoma, mesothelioma, epithelioid atypical Spitz tumors \\
\hline \multicolumn{3}{|l|}{ Papillary Carcinoma } \\
\hline Hereditary Leiomyomatosis & $\mathrm{FH}$ & $\begin{array}{l}\text { Cutaneous and uterine leiomyomata; } \\
\text { Papillary renal carcinoma type } 2\end{array}$ \\
\hline Hereditary Papillary Renal Cancer & MET & Papillary renal carcinoma type 1 \\
\hline \multicolumn{3}{|l|}{ Other Histology } \\
\hline Tuberous Sclerosis Complex & TSC1, TSC2 & $\begin{array}{l}\text { Facial angiofibromas, subependymal giant cell astrocytoma, } \\
\text { subependymal nodules, CNS cortical tubules; renal } \\
\text { angiomyolipoma }\end{array}$ \\
\hline SDH-associated Renal Cancer & $\begin{array}{l}S D H B, S D H C \\
S D H D\end{array}$ & $\begin{array}{l}\text { Paraganglioma, pheochromocytoma; renal clear cell, } \\
\text { chromophobe, or oncocytoma }\end{array}$ \\
\hline Lynch Syndrome & MLH1, MSH2 & $\begin{array}{l}\text { Familial history of cancer, primarily colon endometrial, ovarian, } \\
\text { small bowel, upper urinary tract urothelial carcinoma, pancreatic; } \\
\text { clear cell and papillary renal carcinoma described ( } 25)\end{array}$ \\
\hline
\end{tabular}

\section{Clinical Decision Making}

Genomic testing currently has no established role in the prognosis and treatment of kidney cancer, though some genomic factors are emerging in clear cell carcinoma of the kidney that may contribute to clinical decision making in the future. Clear cell renal cell carcinoma (ccRCC) is characterized by loss or inactivation of the VHL gene (26), located on the short arm of chromosome 3. The development of haplo insufficiency at $3 p$ appears to be an early event in the genetic evolution of ccRCC (27). A second hit to VHL typically occurs later in life via mutation or methylation events that result in a decrease or loss of expression of VHL (28). This results in downstream signaling changes promoting tumorigenesis. Given the early and nearly ubiquitous presence of VHL abnormalities in ccRCC, other genomic markers have been explored as potential prognostic and predictive markers.
Mutations in PBRM1 and BAP1, which are also located on $3 \mathrm{p}$, have been shown to have prognostic utility (29). Expression of PBRM1 and BAP1 from resected ccRCC specimens stratified outcomes into four distinct groups. PBRM1+/BAP1+ specimens had the longest relapse free and disease specific survival, while PBRM-/BAP1- tumors had the worst outcomes. These genetic findings correlated with traditional pathologic characteristics that are associated with poor outcomes, such as tumor size, TNM stage, nuclear grade, and tumor necrosis. While this very nicely ties the genetic abnormalities to the biology and clinical features of the disease, it does not provide a superior methodology for estimating risk.

The MTOR pathway has been a target of treatment in ccRCC, with both temsirolimus and everolimus approved. Mutations in TSC1, TSC2, and MTOR have been demonstrated in ccRCC, with TSC1 muta- 
tions associated with the development of higher grade tumors (30). These mutations have also been associated with response to mTOR inhibitors (31), which have been approved for use in poor risk patients. Mutations in PBRM1 have been associated with response to the immune checkpoint inhibitors, though the strength and mechanism of this interaction is unclear (32).

\section{Summary}

While our understanding of the genomic factors underlying the clinical behavior of ccRCC has made tremendous progress in the last decade, genomic testing in kidney cancer has little role in the treatment of patients outside the context of clinical trials. A deeper understanding of the genomic alterations driving biological behavior is emerging. In patients with a strong family history of syndromes associated with an increased risk of kidney cancer or clinical characteristics of these syndromes, consideration of germline testing should be given and discussed with the patient.

\section{Urothelial Carcinoma}

\section{Genetic Risk}

Bladder cancer has evidence of heritable risk factors, though exact genomic mechanisms of this risk are less well understood. Urothelial carcinoma is associated with hereditary non-polyposis colorectal cancer, also known as the Lynch syndrome. Defects in $\mathrm{MSH} 2$ in particular were associated with an increased risk of developing upper tract urothelial carcinoma in a Danish cohort of families with Lynch syndrome (33). Renal pelvis and ureteral urothelial carcinoma is included in the Amsterdam II criteria as a Lynch syndromeassociated cancer. Patients who present with upper tract disease should undergo detailed family history to explore for evidence of heritable mismatch repair defects and have germline testing performed as appropriate (34).

\section{Clinical Decision Making}

Molecular subtyping of urothelial cancer utilizing gene expression profiling techniques has emerged as a potential prognostic and predictive tool. Four subtypes emerged from the Cancer Genome Atlas project via a hierarchical clustering analysis, originally described as clusters I, II, III, and IV (35). Clusters I and II refer to luminal subtypes and clusters III and IV to basal subtypes. Other groups have divided the subtypes differently, but similarities exist between the various categories. Basal subtypes have been shown to be more aggressive and are associated with worse survival in chemotherapy-naïve urothelial carcinoma (36). Interestingly, basal subtypes have also been shown to have the best outcomes after administration of neoadjuvant chemotherapy (37). Molecular subtyping may also provide predictive information regarding response to immunotherapy. Utilizing the Cancer Genome Atlas (TCGA) subtyping classification, cluster III subtypes had the highest response rate compared with other subtypes at $30 \%$ following treatment with nivolumab in the second line setting (38). Cluster III subtypes were also associated with the strongest interferon- $\gamma$ expression signature, which also correlated with a higher likelihood of response (38). The same TCGA subtyping was utilized in the corresponding clinical trial for atezolizumab following platinum based chemotherapy. Cluster II subtypes showed the best response rate to therapy at $34 \%$. However, PD-L1 expression levels and CD8 T cell gene expression markers were higher in the cluster III subtype (39). These data come from smaller phase II trials and will be explored in larger phase III trials.

In addition to broad molecular subtyping, specific genetic mutations have been identified that may impact clinical decision making. Somatic mutations in the nucleotide excision repair gene ERCC2 are present in $7-12 \%$ of 
urothelial carcinoma $(35,40)$. Mutations in ERCC2 are associated with a distinct molecular subtype (41) that overlaps with luminal subtypes. The presence of ERCC2 mutations was enriched in patients who responded to cisplatin chemotherapy (40) and later validated as a biomarker of platinum sensitivity (42). Mutations in other DNA damage response and repair (DDR) machinery have also been identified in urothelial carcinoma as a biomarker of response to immunotherapy. In a series of 60 patients, $46.7 \%$ had an alteration in DDR genes with $25 \%$ having deleterious alterations (43). Deleterious DDR mutations were found in ATM, POLE, BRCA2, ERCC2, FANCA, and MSH6. The presence of DDR mutations were associated with higher response rates, longer progressive free survival, and longer overall survival with immunotherapy. Mutations in FGFR3 have been identified as an intriguing target for therapy. Cluster I subtypes are enriched with FGFR3 mutations (35) and are generally associated with immunologically cold tumors that do not respond to checkpoint inhibitors $(38,39)$. Small molecule tyrosine kinase inhibitors of FGFR3 are currently in development and showing promising results. The FGFR3 TKI erdafitinib was recently reported in a phase II trial to have an overall response rate of $40 \%$ (44). Given that this is a population that has poorer responses to immunotherapy and minimal therapeutic options, erdafitinib represents a potential breakthrough for patients with urothelial cancer.

\section{Summary}

Urothelial carcinoma has some association with the Lynch syndrome, especially when presenting with upper urinary tract disease, and a detailed family history should be obtained in these patients. Referral to genetic counselor may be warranted if history suggests the presence of a heritable syndrome. Molecular profiling of urothelial carcinoma has revealed distinct subgroups of disease with differing clinical behavior. There are early signs that molecular subtyping may play a role in prognosis and treatment selection (Table 3), but this is currently not applicable outside the clinical trial setting. ERCC2 mutations are useful in predicting response to platinum chemotherapy, are not specific enough to warrant omitting administration of cisplatin to patients who are oth-

Table 3. Urothelial Cancer Summary

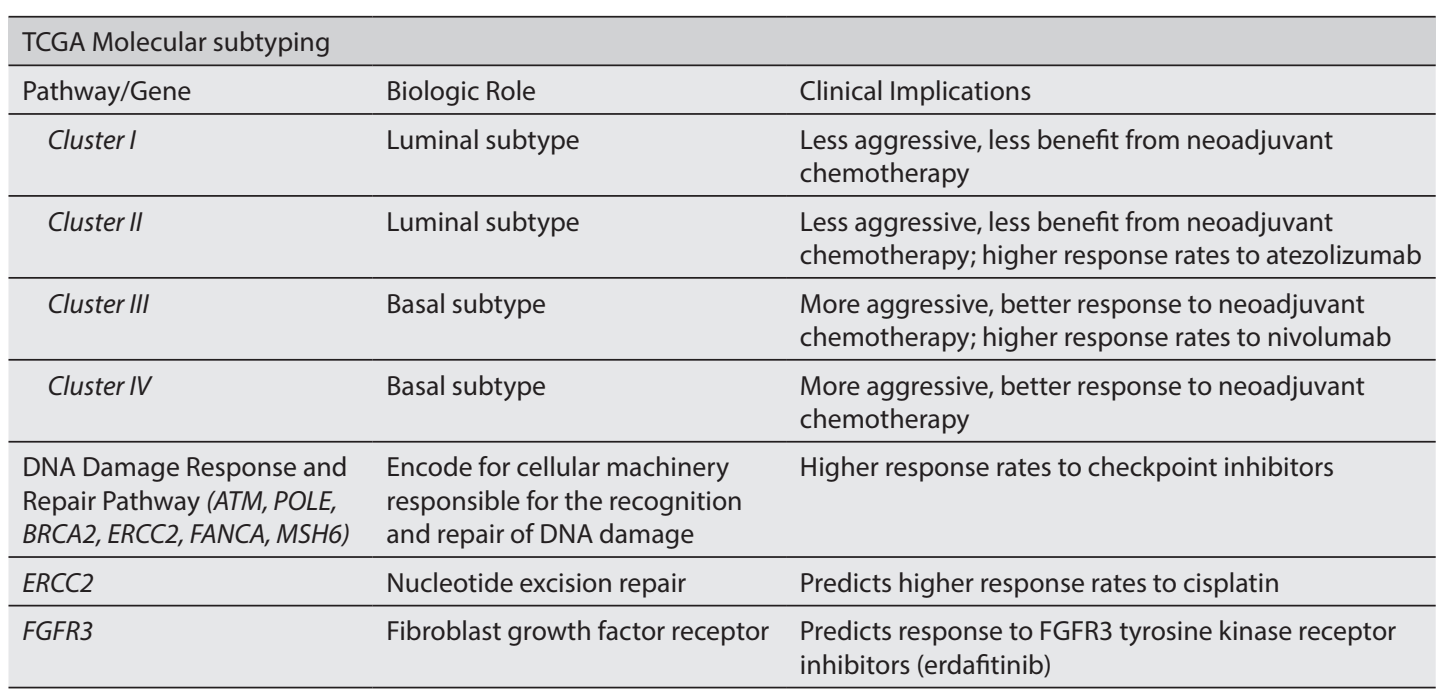

TCGA=The Cancer Genome Atlas. 
erwise eligible. This is also true of defects in DDR genes as they relate to administration of checkpoint inhibitors. FGFR3 alterations will likely become a key piece of information, as targeted therapy is likely to be approved for patients with these alterations in the near future pending completion of phase III studies.

\section{Conclusions}

Clinical breakthroughs resulting from a deeper understanding of the genetic influences in cancer have been a leap forward in the field of oncology. Genitourinary malignancies have not felt the impact as significantly. Breast and thoracic malignancies are prime examples of the potential power of genomic data, as genomic data influences decisions on treatment in a large proportion of patients. Genomic data is currently most relevant in identifying patients carrying high risk germline alterations for genitourinary cancers. The role of genomic testing to influence treatment decisions is currently limited. Fortunately, the field is changing rapidly and breakthroughs driven by genomic information are on the horizon.

Authors' Contributions: Conception and design: MED and RD; Acquisition, analysis and interpretation of data: MED; Drafting the article: MED; Revising it critically for important intellectual content: MED and $\mathrm{RD}$; Approved final version of the manuscript: MED and RD.

Conflict of Interest: Dr. Devitt has received compensation as advisor/speaker for Bayer Pharmaceuticals. Dr. Dreicer has received compensation as adviser for Incyte, Astra Zeneca, Seattle Genetics, EMD Serono, Pfizer.

\section{References}

1. Mucci LA, Hjelmborg JB, Harris JR, Czene K, Havelick DJ, Scheike T, et al. Familial Risk and Heritability of Cancer Among Twins in Nordic Countries. JAMA. 2016 Jan 5;315(1):68-76. Erratum in: JAMA. 2016 Feb 23;315(8):822.
2. Bratt O, Drevin L, Akre O, Garmo H, Stattin P. Family history and probability of prostate cancer, differentiated by risk category: A nationwide population-based study. J Natl Cancer Inst. 2016;108(10).

3. Ewing CM, Ray AM, Lange EM, Zuhlke KA, Robbins CM, Tembe WD, et al. Germline mutations in HOXB13 and prostate-cancer risk. N Engl J Med. 2012;366(2):141-9.

4. Huang H, Cai B. G84E mutation in HOXB13 is firmly associated with prostate cancer risk: A meta-analysis. Tumour Biol. 2014;35(2):1177-82.

5. Giri VN, Beebe-Dimmer JL. Familial prostate cancer. Semin Oncol. 2016;43(5):560-5.

6. Agalliu I, Karlins E, Kwon EM, Iwasaki LM, Diamond A, Ostrander EA, et al. Rare germline mutations in the BRCA2 gene are associated with earlyonset prostate cancer. Br J Cancer. 2007;97(6):826-31.

7. Edwards SM, Kote-Jarai Z, Meitz J, Hamoudi R, Hope Q, Osin P, et al. Two percent of men with early-onset prostate cancer harbor germline mutations in the BRCA2 gene. Am J Hum Genet. 2003;72(1):1-12.

8. Gallagher DJ, Gaudet MM, Pal P, Kirchhoff T, Balistreri L, Vora K, et al. Germline BRCA mutations denote a clinicopathologic subset of prostate cancer. Clin Cancer Res. 2010;16(7):2115-21.

9. Castro E, Goh C, Olmos D, Saunders E, Leongamornlert D, Tymrakiewicz M, et al. Germline BRCA mutations are associated with higher risk of nodal involvement, distant metastasis, and poor survival outcomes in prostate cancer. J Clin Oncol. 2013;31(14):1748-57.

10. Dominguez-Valentin M, Joost P, Therkildsen C, Jonsson M, Rambech E, Nilbert M. Frequent mismatch-repair defects link prostate cancer to lynch syndrome. BMC Urol. 2016;16:15.

11. Benafif S, Kote-Jarai Z, Eeles RA, PRACTICAL Consortium. A review of prostate cancer genomewide association studies (GWAS). Cancer Epidemiol Biomarkers Prev. 2018;27(8):845-57.

12. National Comprehensive Cancer Network. NCCN guidelines genetic/familial high-risk assessment: Breast and ovarian. version 2.2019. [updated 2018 Jul 30; cited 2018 Aug 10]. Available from: https:// www.nccn.org/professionals/physician_gls/pdf/ genetics_screening.pdf.

13. Robinson D, Van Allen EM, Wu YM, Schultz N, Lonigro RJ, Mosquera JM, et al. Integrative clinical genomics of advanced prostate cancer. Cell. 2015;161(5):1215-28.

14. Antonarakis ES, Lu C, Wang H, Luber B, Nakazawa M, Roeser JC, et al. AR-V7 and resistance to 
enzalutamide and abiraterone in prostate cancer. N Engl J Med. 2014;371(11):1028-38.

15. Armstrong A, Halabi S, Luo J, Nanus DM, Giannakakou P, Zelig Szmulewitz R, et al. The PROPHECY trial: Multicenter prospective trial of circulating tumor cell (CTC) AR-V7 detection in men with $\mathrm{mCRPC}$ receiving abiraterone $(\mathrm{A})$ or enzalutamide (E). J Clin Oncol. 2018;36(15 Suppl):5004.

16. Nakazawa M, Lu C, Chen Y, Paller CJ, Carducci MA, Eisenberger MA, et al. Serial blood-based analysis of AR-V7 in men with advanced prostate cancer. Ann Oncol. 2015;26(9):1859-65.

17. Pritchard CC, Offit K, Nelson PS. DNA-repair gene mutations in metastatic prostate cancer. $\mathrm{N}$ Engl J Med. 2016;375(18):1804-5.

18. Nicolosi P, Michalski S, Freschi B, O’Leary E, Quintana R, Wilson I. Need for re-evaluation of current guidelines based on results from germline genetic testing in prostate cancer. J Clin Oncol. 2017;35(15):5009.

19. Abida W, Cheng WL, Armenia J, Middha S, Autio KA, Rathkopf DE, et al. Microsatellite instability in prostate cancer and response to immune checkpoint blockade. J Clin Oncol. 2018;36(15 Suppl):5020.

20. Pomerantz MM, Spisák S, Jia L, Cronin AM, Csabai I, Ledet E, et al. The association between germline BRCA2 variants and sensitivity to platinumbased chemotherapy among men with metastatic prostate cancer. Cancer. 2017;123(18):3532-9.

21. Mateo J, Carreira S, Sandhu S, Miranda S, Mossop H, Perez-Lopez R, et al. DNA-repair defects and olaparib in metastatic prostate cancer. N Engl J Med. 2015;373(18):1697-708.

22. Armstrong AJ, Halabi S, Healy P, Alumkal JJ, Winters C, Kephart J, et al. Phase II trial of the PI3 kinase inhibitor buparlisib (BKM-120) with or without enzalutamide in men with metastatic castration resistant prostate cancer. Eur J Cancer. 2017;81:228-36.

23. Mateo J, Ganji G, Lemech C, Burris HA, Han SW, Swales K, et al. A first-time-in-human study of GSK2636771, a phosphoinositide 3 kinase betaselective inhibitor, in patients with advanced solid tumors. Clin Cancer Res. 2017;23(19):5981-92.

24. Therkildsen C, Joost P, Lindberg LJ, Ladelund S, Smith-Hansen L, Nilbert M. Renal cell cancer linked to lynch syndrome: Increased incidence and loss of mismatch repair protein expression. Int J Urol. 2016;23(6):528-9.

25. Sato Y, Yoshizato T, Shiraishi Y, Maekawa S, Okuno Y, Kamura T, et al. Integrated molecular analysis of clear-cell renal cell carcinoma. Nat Genet. 2013;45(8):860-7.
26. Gerlinger M, Horswell S, Larkin J, Rowan AJ, Salm MP, Varela I, et al. Genomic architecture and evolution of clear cell renal cell carcinomas defined by multiregion sequencing. Nat Genet. 2014;46(3):225-33.

27. Mitchell TJ, Turajlic S, Rowan A, Nicol D, Farmery JHR, O'Brien T, et al. Timing the landmark events in the evolution of clear cell renal cell cancer: TRACERx renal. Cell. 2018;173(3):611-23.e17.

28. Joseph RW, Kapur P, Serie DJ, Parasramka M, Ho $\mathrm{TH}$, Cheville JC, et al. Clear cell renal cell carcinoma subtypes identified by BAP1 and PBRM1 expression. J Urol. 2016;195(1):180-7.

29. Kucejova B, Peña-Llopis S, Yamasaki T, Sivanand S, Tran TA, Alexander S, et al. Interplay between pVHL and mTORC1 pathways in clear-cell renal cell carcinoma. Mol Cancer Res. 2011;9(9):125565.

30. Kwiatkowski DJ, Choueiri TK, Fay AP, Rini BI, Thorner AR, de Velasco G, et al. Mutations in TSC1, TSC2, and MTOR are associated with response to rapalogs in patients with metastatic renal cell carcinoma. Clin Cancer Res. 2016;22(10):2445-52.

31. Miao D, Margolis CA, Gao W, Voss MH, Li W, Martini DJ, et al. Genomic correlates of response to immune checkpoint therapies in clear cell renal cell carcinoma. Science. 2018;359(6377):801-6.

32. Joost P, Therkildsen C, Dominguez-Valentin M, Jonsson $M$, Nilbert M. Urinary tract cancer in lynch syndrome; increased risk in carriers of MSH2 mutations. Urology. 2015;86(6):1212-7.

33. National Comprehensive Cancer Network. NCCN guidelines genetic/familial high risk assessment: Colorectal. version 1.2018. [updated 2018 Jul 12; cited 2018 Aug 14]. Available from: https://www. nccn.org/professionals/physician_gls/pdf/genetics_colon.pdf.

34. The Cancer Genome Atlas,Research Network, Weinstein JN, Akbani R, et al. Comprehensive molecular characterization of urothelial bladder carcinoma. Nature. 2014;507:315.

35. Choi W, Porten S, Kim S, Willis D, Plimack ER, Hoffman-Censits J, et al. Identification of distinct basal and luminal subtypes of muscle-invasive bladder cancer with different sensitivities to frontline chemotherapy. Cancer Cell. 2014;25(2):15265.

36. McConkey DJ, Choi W, Shen Y, Lee IL, Porten $\mathrm{S}$, Matin SF, et al. A prognostic gene expression signature in the molecular classification of chemotherapy-naive urothelial cancer is predictive of clinical outcomes from neoadjuvant chemotherapy: A phase 2 trial of dose-dense metho- 
trexate, vinblastine, doxorubicin, and cisplatin with bevacizumab in urothelial cancer. Eur Urol. 2016;69(5):855-62.

37. Sharma P, Retz M, Siefker-Radtke A, Baron A, Necchi A, Bedke J, et al. Nivolumab in metastatic urothelial carcinoma after platinum therapy (CheckMate 275): A multicentre, single-arm, phase 2 trial. Lancet Oncol. 2017;18(3):312-22.

38. Rosenberg JE, Hoffman-Censits J, Powles T, van der Heijden MS, Balar AV, Necchi A, et al. Atezolizumab in patients with locally advanced and metastatic urothelial carcinoma who have progressed following treatment with platinum-based chemotherapy: A single-arm, multicentre, phase 2 trial. Lancet. 2016;387(10031):1909-20.

39. Van Allen EM, Mouw KW, Kim P, Iyer G, Wagle $\mathrm{N}, \mathrm{Al}$-Ahmadie H, et al. Somatic ERCC2 mutations correlate with cisplatin sensitivity in muscleinvasive urothelial carcinoma. Cancer Discov. 2014;4(10):1140-53.

40. Kim J, Mouw KW, Polak P, Braunstein LZ, Kamburov A, Kwiatkowski DJ, et al. Somatic ERCC2 mutations are associated with a distinct genomic signature in urothelial tumors. Nat Genet. 2016;48(6):600-6.

41. Liu D, Plimack ER, Hoffman-Censits J, Garraway LA, Bellmunt J, Van Allen E, et al. Clinical validation of chemotherapy response biomarker ERCC2 in muscle-invasive urothelial bladder carcinoma. JAMA Oncol. 2016;2(8):1094-6.

42. Teo MY, Bambury RM, Zabor EC, Jordan E, AlAhmadie H, Boyd ME, et al. DNA damage response and repair gene alterations are associated with improved survival in patients with platinumtreated advanced urothelial carcinoma. Clin Cancer Res. 2017;23(14):3610-8.

43. Siefker-Radtke AO, Necchi A, Hoon Park S, Garcia-Donas J, Huddart RA, Burgesset EF, al. First results from the primary analysis population of the phase 2 study of erdafitinib (ERDA; JNJ-42756493) in patients (pts) with metastatic or unresectable urothelial carcinoma (mUC) and FGFR alterations (FGFRalt). J Clin Oncol. 2018;36(15 Suppl):4503. 